\title{
Enhanced post-ischemic neurogenesis in aging rats
}

\author{
Yao-Fang Tan ${ }^{1}$, Edward Preston ${ }^{2}$ and J. Martin Wojtowicz ${ }^{\text {* }}$
}

1 Department of Physiology, University of Toronto, Toronto, ON, Canada

2 Institute of Biological Sciences, National Research Council, Ottawa, ON, Canada

\section{Edited by:}

Gerd Kempermann, Center for

Regenerative Therapies, Germany

\section{Reviewed by:}

Ashok K. Shetty, Duke University Medical Center, USA

Sebastian Jessberger, Swiss Federal Institute of Technology Zurich,

Switzerland

Alejandro F. Schinder, Leloir Institute,

Argentina

\section{${ }^{*}$ Correspondence:}

J. Martin Wojtowicz, Department of Physiology, University of Toronto, 1 King's College Circle, Room 3214, Toronto, ON, Canada M5S 1 A8.

e-mail:martin.wojtowicz@utoronto.ca
Hippocampal neurogenesis persists in adult mammals, but its rate declines dramatically with age. Evidence indicates that experimentally-reduced levels of neurogenesis (e.g., by irradiation) in young rats has profound influence on cognition as determined by learning and memory tests. In the present study we asked whether in middle-aged, 10- to 13-months-old rats, cell production can be restored toward the level present in young rats. To manipulate neurogenesis we induced bilateral carotid occlusion with hypotension. This procedure is known to increase neurogenesis in young rats, presumably in a compensatory manner, but until now, has never been tested in aging rats. Cell production was measured at 10,35, and 90 days after ischemia. The results indicate that neuronal proliferation and differentiation can be transiently restored in middle-aged rats. Furthermore, the effects are more pronounced in the dorsal as opposed to ventral hippocampus thus restoring the dorso-ventral gradient seen in younger rats. Our results support previous findings showing that some of the essential features of the age-dependent decline in neurogenesis are reversible. Thus, it may be possible to manipulate neurogenesis and improve learning and memory in old age.

\section{Keywords: hippocampus, adult neurogenesis, ischemia, stroke, dentate gyrus, aging}

\section{INTRODUCTION}

The functional role of adult neurogenesis in the hippocampal dentate gyrus remains controversial. Dentate gyrus as a whole is thought to be strongly related to cognitive decline in old age but the exact contribution of neurogenesis vs. other forms of plasticity is uncertain (Small et al., 2004; Burke and Barnes, 2006). There is convincing evidence that experimentally-induced decline in neurogenesis produces severe impairments in performance on some but not all memory tasks (Deng et al., 2010). Studies attempting to correlate natural decline of neurogenesis and memory with age have been controversial. In one such study, a sub-population of old (20 months) rats showed cognitive impairment in correlation with the number of adult-produced new neurons (Drapeau et al., 2003). Interestingly, old unimpaired rats performed nearly as well as the young ( 2 months) rats even though the former had only a fraction of the new neurons in comparison to the young. Other studies failed to demonstrate a relationship between neurogenesis and memory performance (Bizon and Gallagher, 2003; Merrill et al., 2003). Methodological reasons may account for these discrepancies between studies. For example, it would appear that spatial memory tasks may not be optimal for testing the influence of neurogenesis since many studies failed to demonstrate a clear cause-effect relationship in such tasks (Leuner et al., 2006) while others produced varied effects on either short-term or long-term memory (Dupret et al., 2008; Imayoshi et al., 2008; Jessberger et al., 2009). Species differences should also be considered since neurogenesis varies in its rate and physiological significance among species (Snyder et al., 2009a; Johnson et al., 2010).

Emerging evidence indicates that memory tasks involving detection and processing of context are most sensitive to changes in neurogenesis and should be good indicators of deteriorating memory performance at old age (Wojtowicz et al., 2008). Thus, a working hypothesis suggests that aging animals can adapt to low levels of neurogenesis, but fundamental dependence of memory on new neurons persists in old age. It is therefore of interest to test whether rate of neurogenesis can be manipulated in old animals. Studies by Kempermann et al. $(1998,2002)$ have shown that neurogenesis in middle-aged mice is responsive to stimulation by enriched environment and the increases are correlated with modest improvements in learning. In addition, other forms of physiological and pathological stimulation can enhance neurogenesis in aging animals albeit often to a limited extent (Cameron and McKay, 1999; van Praag et al., 2005; Hattiangady et al., 2008).

Changes in neurogenesis are also of interest with regard to possible recovery from ischemic damage. The discovery by Liu et al. (1998), showing that global ischemia is followed by increase in hippocampal neurogenesis, opened up a possibility that neurogenesis may be a compensatory, adaptive mechanism that could represent functional recovery after stroke or injury. Since adult neurogenesis is often mentioned as an ultimate mechanism of brain repair after injury (Kokaia and Lindvall, 2003; Lichtenwalner and Parent, 2006) we explore the extent of post-ischemic neurogenesis in middle-aged rats that may be representative of the aging, stroke-prone human population. Injury-triggered neurogenesis has been observed after global or focal ischemia in a variety of animal models (Sharp et al., 2002; Lichtenwalner and Parent, 2006). Yagita et al. (2001) reported increased proliferation, but reduced survival of new neurons following global ischemia in middle-aged Wistar rats in comparison to young adults. (Darsalia et al., 2005) claim reduced post-ischemic neurogenesis in dentate gyrus of 15-months-old Wistar rats but in parallel with retained enhancement of neurogenesis in the subventricular zone. Jin et al. (2004) have found blunted neurogenesis in old (24 months) Fisher 344 rats following a focal ischemia. In the present study we have used the two vessel occlusion (2VO) 
with hypotension in middle-aged male S-D rats in comparison to our previous study on young adult (3 months) animals (Kee et al., 2001).

\section{MATERIALS AND METHODS ANIMAL GROUPS}

Thirty 10-months-old male Sprague-Dawley rats weighing $522 \pm 50 \mathrm{~g}$ were obtained from Harlan (Indianapolis, IN, USA). At 10 months, Sprague-Dawley rats are considered not yet senescent but rather "middle-aged". Previous studies found that the main effects of age on neurogenesis were evident at this time point (McDonald and Wojtowicz, 2005). The animals were allowed to acclimatize for 1 week prior to surgery and divided into the following groups: 10 days sham, 10 day ischemic, 35 day sham, 35 day ischemic $(n=6$ each), 90 days sham and 90 days ischemic ( $n=3$ each).

\section{THE 2-VESSEL OCCLUSION WITH HYPOTENSION (2V0)}

Cerebral ischemia in rats was produced by our adaptation of the $2 \mathrm{VO}$ method of (Smith et al., 1984) and as described previously (Preston and Webster, 2004). Ischemia was effected for $12 \mathrm{~min}$ by combining bilateral carotid artery occlusion with hypovolemic hypotension to minimize compensatory vertebral blood flow. Each rat was anesthetized with sodium pentobarbital (65 mg/kg i.p.), intubated using a pediatric laryngoscope, and mechanically ventilated (Harvard rodent ventilator, Ealing Scientific, St. Laurent PQ) with a 30:70 mixture of $\mathrm{O}_{2}$ and $\mathrm{N}_{2}$. delivered at 35-40 breaths/min, $3 \mathrm{ml}$ volume. Tympanic and colonic temperatures were measured using YSI 511 and 402 thermistor probes (Yellow Springs Instruments, Yellow Springs, $\mathrm{OH}$, USA) and were maintained close to $37.5 \pm 0.5\left({ }^{\circ} \mathrm{C}\right)$ by means of a circulating water pad under the supine rat. The tail artery was cannulated and arterial blood sampled to measure blood gases and $\mathrm{pH}$. Ventilation rate was adjusted if required to obtain physiologically normal blood gas parameters (Preston and Webster, 2004). The common carotid arteries were exposed and freed for placement of vascular clamps. Arterial pressure was monitored by a polygraph (Biopac Systems, Galeta, CA, USA) and Statham pressure transducer hooked by a Y-connection to the tail artery catheter. Pressure was lowered by withdrawing $7-8 \mathrm{ml}$ of tail artery blood into a $10 \mathrm{ml}$ heparinized syringe. Both common carotid arteries were then occluded by vascular clamps for $12 \mathrm{~min}$ during which time arterial pressure was maintained between $42-47 \mathrm{mmHg}$ by adjusting the volume of blood in the syringe. The blood was then returned to the rat, the incisions closed with sutures, and mechanical ventilation withdrawn. Colonic temperature monitoring was continued and normothermia maintained in a lamp-warmed cage until the rat recovered from anesthesia. Sham-operated animals underwent an identical procedure, including lowering blood pressure to $42-47 \mathrm{mmHg}$ for $12 \mathrm{~min}$, except that the carotid arteries were not clamped. This group served as a control to rule out possible factors other than ischemia that might alter the recovery, such as stress resulting from handling, surgery, change in blood pressure, and transportation to another laboratory for immunohistochemical procedures.

Principles of laboratory animal care were followed and animal care committees at the University of Toronto approved the procedures. The aged rats withstood the $2 \mathrm{VO}$ procedure as well as the young rats did in past studies. There was no excessive mortality associated with the use of the aging animals.

\section{BrdU ADMINISTRATION}

5-Bromo-2-deoxyuridine (BrdU; Sigma, St. Louis, MO, USA) was dissolved in phosphate-buffered saline (PBS) containing $10 \mathrm{mM}$ $\mathrm{NaOH}$ for a final concentration of $20 \mathrm{mg} / \mathrm{ml}$. The solution was injected i.p. at the dose of $200 \mathrm{mg} / \mathrm{kg}$ of body weight at $9 \mathrm{AM}$ and again at $9 \mathrm{PM}$ on the seventh day after the surgery (2VO or sham). Thus, each rat received $400 \mathrm{mg}$ BrdU/kg. This dose was chosen to allow maximal labeling of cells born over the 24-h period according to the procedure described by McDonald and Wojtowicz (2005). A single injection of BrdU is preferred over repeated injections, since it allows precise birthing of the new cells. Due to the 12-h separation, approximately equivalent to the duration of the $S$-phase, between the injections no cells should be labeled twice.

\section{IMMUNOHISTOCHEMISTRY}

Following intracardiac perfusion with PBS and 4\% paraformaldehyde, the right hippocampus was sectioned into three parts - dorsal, middle and ventral - and fixed overnight in $4 \%$ paraformaldehyde at $4^{\circ} \mathrm{C}$. The left hippocampus was used for preliminary electrophysiological experiments (not reported here). Serial $30 \mu \mathrm{m} \mathrm{sec}-$ tions of all segments were cut with a vibratome resulting in a total of 200-250 sections per animal. One in ten sections were sampled using a systematic random sampling procedure along the whole dorso-ventral length of the hippocampus. In animals killed at the 90 days time point the sampling frequency was doubled to reach sufficient sampling accuracy. For all groups of animals the criteria for sampling were those outlined by West et al. (1991) and recently reviewed by Wojtowicz and Kee (2006). Thus, for BrdU estimates (the worst case scenario with very few cells to count) in sham control animals for example, $\mathrm{CE}$ among the sections was generally below 0.10 , while the variance $(\mathrm{CV})^{2}$ among the animals was always $>0.20$. Immunohistochemical procedures for detection of neuronal nucleus (NeuN), doublecortin (DCX), BrdU, and Calbindin ( $\mathrm{CaBP})$ were the same as described previously, using the same antibodies from the same suppliers (Wang et al., 2005; Wojtowicz and Kee, 2006). To label for a microglial marker ED1 (CD68) we incubated sections with a primary antibody (mouse, anti-rat, Chemicon) for $24 \mathrm{~h}$ at $4^{\circ} \mathrm{C}$ at 1:200. Washed and incubated with a secondary antibody (goat, IgG, $488 \mathrm{~nm}$, Molecular probes) at 1:200 for $2 \mathrm{hrs}$ at room temperature. A fluorescent marker FluoroJade B (Chemicon) was used to stain for degenerating neurons as described by Schmued and Hopkins (2000).

Immunopositive cells (DCX and BrdU) were counted in a defined region of hippocampus. Specifically, cells were counted if they resided in the subgranular zone (SGZ) of the dentate gyrus and in granule cell layer (GCL). The SGZ was defined as a two-cell width wide (approximately $20 \mu \mathrm{m}$ ) region just below the GCL. All cells within each section were counted, but excluding top and bottom surfaces of the sections in order to avoid counting cells that were dissected or damaged. The average number of cells per section was multiplied by the total number of sections to obtain total cell numbers per DG. In some cases, where we compare dorsal vs. ventral regions, we also report average cell densities per section for each region. Double-labeling (DCX/BrdU and $\mathrm{CaBP} / \mathrm{BrdU}$ ) was identified using a Leica TCS-SL confocal microscope (Leica Microsystems (Canada) Inc.; Richmond Hill, ON, Canada) with a $63 \times$ objective lens. 


\section{NeUN AREA MEASUREMENTS AND STATISTICAL ANALYSIS}

To measure the NeuN+ areas, six sections from the three representative animals in each of the main time groups, i.e., 10, 35, and 90 days were selected. The stained sections were digitized and the areas covered by DG and CA1 were outlined using a cursor. The same criteria for threshold detection were used for all 36 sections ( 9 dorsal 2VO, 9 ventral 2VO, 9 dorsal sham and 9 ventral sham). The area measurements were obtained using Image J software (Http:// rsb.info.nih.gov/ij/). Mean values of these areas are presented in Table 1, giving reliable, comparative estimates of the neuronal populations in CA1 and DG of $2 \mathrm{VO}$ and sham animals.

Statistical analysis was performed with SigmaStat software. Data sets from control and 2VO groups were compared using 2-way ANOVA with time and treatment as the two variables and posthoc pairwise comparisons with Holm-Sidak method at $P<0.05$. In some cases where only two groups were compared at a single time point a $t$-test or equivalent non-parametric test were used.

\section{RESULTS}

The $2 \mathrm{VO}$ procedure has not been applied to middle-aged rats so we opted to characterize its effects on neuronal survival in the hippocampus. We used NeuN immunohistochemistry to illustrate neurons and measured the areas covered by the neuronal profiles within the CA1 field and the DG (Figures 1A-D).

As reported previously for young adults, the $2 \mathrm{VO}$ ischemia had a selective effect on neuronal death in dorsal CA1 but spared the DG (Kee et al., 2001). To support the evidence that loss of NeuN staining is caused by ischemia we also stained for marker of neuronal degeneration FluoroJade B and marker of inflammation, ED1. Both of these markers showed strong positive staining in CA1, particularly in the dorsal region, but not in DG (Figures 1E-H).

Table 1 includes detailed measurements of NeuN+ cell layers in CA1 and DG at 10, 35, and 90 days, following the ischemia. Only the CA1 fields showed significant decrease in the stained areas suggesting loss of neurons. The neuronal loss, as measured by NeuN staining, was almost complete (99\%) in the dorsal CA1 and less severe (20-50\%) in the ventral region. All these results confirm that $2 \mathrm{VO}$ procedure in 1-year-old animals had effects on neuronal survival and death that were similar to previously observed effects in young (3-months old) adults (Kee et al., 2001; Wang et al., 2005).
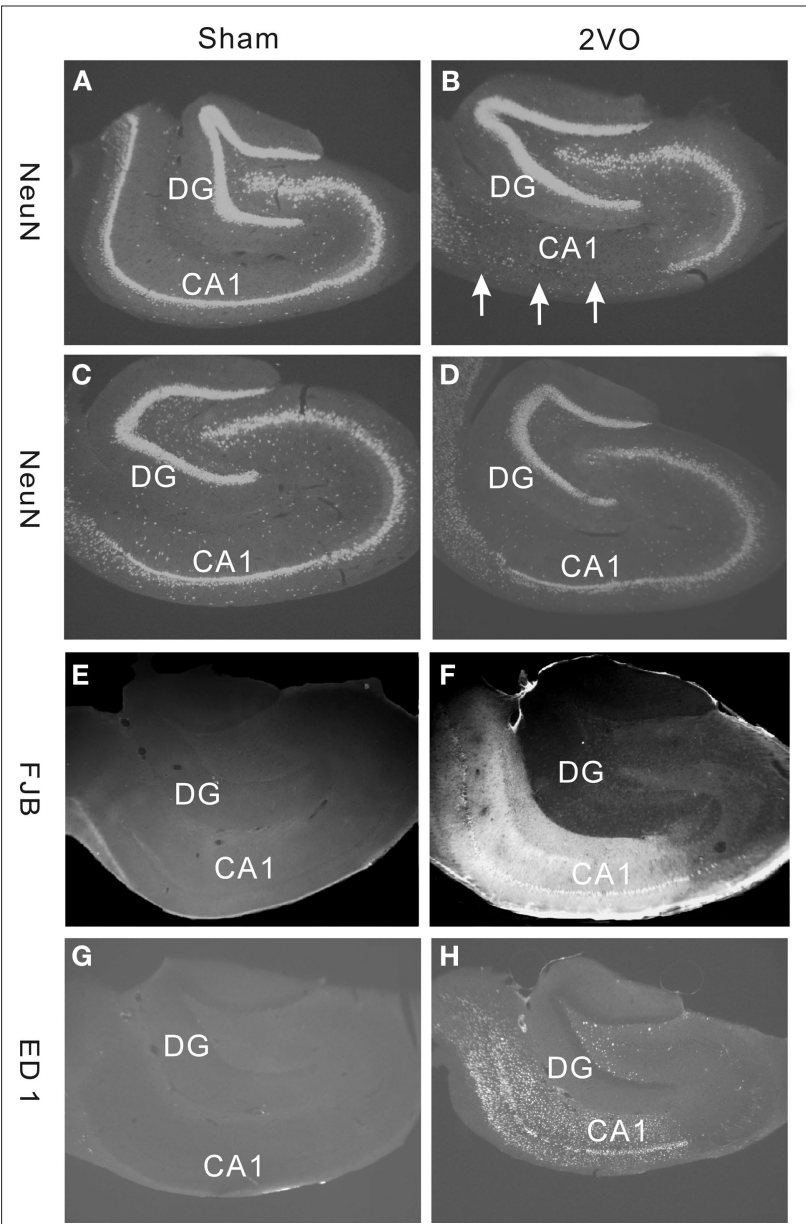

FIGURE 1 | Immunohistochemical staining demonstrating effects of sham (control) and 2VO (ischemia) procedure in dorsal and ventral regions of the hippocampus. (A-D) Show sections stained with a neuronal marker NeuN. Note, a severe loss of cells in dorsal CA1 field (arrows) but no obvious cell loss in DG (A,B). Much lesser effect is seen in ventral CA1 (C,D) $(\mathbf{E}, \mathbf{F})$ Staining with a neuronal degeneration marker Fluorojade B (FJB) shows clear degeneration in $\mathrm{CA} 1$ field in dorsal region. $\mathbf{( G , H )}$ Staining with a microglia marker ED1 shows strong inflammatory response in CA1 and Hilus but not in DG. Sections in (A-D) are from representative animals within the 35 day group. Sections in (E-H) are from animals within the 10 day group.

Table 1 | Summary of NeuN+ area measurements indicating regional changes in hippocampal cell layers at different time points after ischemia.

\begin{tabular}{|c|c|c|c|c|c|c|c|c|c|}
\hline \multirow[b]{2}{*}{ Area } & \multicolumn{3}{|c|}{10 days } & \multicolumn{3}{|c|}{35 days } & \multicolumn{3}{|c|}{90 days } \\
\hline & Sham & $2 \mathrm{VO}$ & $\% \Delta$ & Sham & $2 \mathrm{VO}$ & $\% \Delta$ & Sham & $2 \mathrm{VO}$ & $\% \Delta$ \\
\hline & $\pm 19,920$ & $\pm 4,500$ & & $\pm 30,410$ & $\pm 29,830$ & & $\pm 24,240$ & $\pm 50,390$ & \\
\hline \multirow[t]{2}{*}{ DG Ventral } & 193,100 & 191,100 & $-1.4 \%$ & 178,800 & 200,300 & $-12 \%$ & 200,800 & 202,700 & $\pm 1 \%$ \\
\hline & $\pm 9,840$ & $\pm 27,360$ & & $\pm 12,290$ & $\pm 11,870$ & & $\pm 19,320$ & $\pm 7,410$ & \\
\hline & $\pm 20,160$ & \pm 725 & & $\pm 9,410$ & $\pm 1,120$ & & $\pm 7,240$ & $\pm 12,950$ & \\
\hline \multirow[t]{2}{*}{ CM Ventral } & 56,900 & $45,600^{*}$ & $-20 \%$ & 83,700 & $38,600^{*}$ & $-54 \%$ & 68,900 & $39,800 *$ & $-42 \%$ \\
\hline & $\pm 7,640$ & $\pm 13,790$ & & $\pm 12,760$ & $\pm 5,340$ & & $\pm 6,570$ & $\pm 26,900$ & \\
\hline
\end{tabular}

Mean areas $\left(\mu \mathrm{m}^{2}\right)$ and standard deviations are shown for each group. *indicates statistically significant difference between sham and 2 VO groups. 2 way ANOVA with $n=3$ in each group taking total time and treatment as variables. Area measurements $\left(\mu \mathrm{m}^{2}\right)$ were done using Image $\mathrm{J}$ software as detailed in methods. 
To measure new cell production in DG we injected BrdU on day 7 after the ischemia and perfused animals on days 10,35, and 90. Day 7 was chosen on the basis of past experiments showing the peak of post-ischemic cell production at that time point (Liu et al., 1998; Sharp et al., 2002). The three post-BrdU survival intervals allow for measurements of net cell proliferation, neuronal differentiation, maturation and long-term survival. On the post-ischemic days 10, 35, and 90 the numbers of BrdU+ cells were increased by $240 \%, 960 \%$ and $590 \%$, respectively (Figure 2, Table 2).

An independent measurement of the total number of cells expressing a young neuronal marker DCX showed significant increases on days 10 and 35 but not on day 90 (Figure 3 and Table 2).

Thus, the post-ischemic increase in neurogenesis was large but transient. Double-labeling experiments demonstrated expression pattern of immature neuronal marker (DCX/BrdU) and mature neuronal marker $(\mathrm{CaBP} / \mathrm{BrdU})$ to be similar in control and ischemic groups (Table 2). Considering large absolute

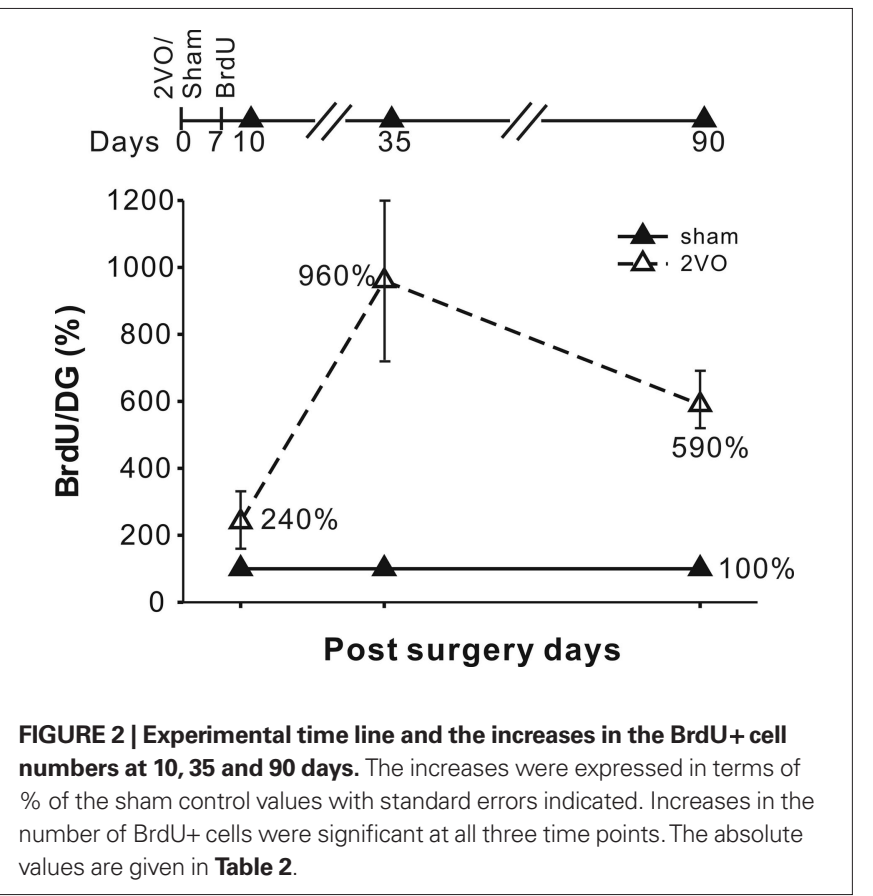

numbers of neuronal precursors generated after ischemia this represents substantial net addition of potential new neurons in ischemic animals.

To further evaluate functionality of neurogenesis after ischemia we examined dorso-ventral distribution of the new neuron density. It has been previously reported that adult neurogenesis in young animals is most pronounced in the dorsal (or septal) hippocampal region as opposed to the ventral (or caudal) region (Snyder et al., 2008). In older animals this gradient is diminished (Snyder et al., 2009b) and there is a corresponding shift in responsiveness of new neurons to physiological stimuli, such as running, toward the ventral region (Snyder et al., 2009b). Our results confirm that there was no dorso-ventral gradient in the density of DCX+ cells in control, aging animals. The numbers of DCX+ young neurons in the dorsal third and ventral third of the hippocampus on day 35 were $7.6 \pm 3.3(\mathrm{SD}) /$ section and $8.6 \pm 2.2(\mathrm{SD}) /$ section, respectively $(P=0.61, t$-test, N.S.). These values were increased to $26.6 \pm 3(\mathrm{SD}) /$ section and $13.7 \pm 4.4(\mathrm{SD}) /$ section after ischemia $(P=0.014, t$-test $)$ thus restoring the twofold dorso-ventral difference that is normally seen in young animals.

\section{DISCUSSION}

The present study has been modeled after previous work by Kee et al. (2001), Wang et al. (2005), but instead of using 3-months-old rats we opted for 10-months old. At this age adult neurogenesis is much reduced although the rate of decline varies with rat strain and living conditions (McDonald and Wojtowicz, 2005; Rao et al., 2006; Epp et al., 2009). Consequently, the basal numbers of DCX+ cells were in the 1,200-1,800 range instead of $>6,000$ usually seen at 3 months (Wojtowicz, 2008). These lower levels of neurogenesis may be adaptive and old animals can perform at or near the performance levels of the young ones (Drapeau et al., 2003; Johnson et al., 2010). However, the critical tests of correlating neurogenesis with memory on tasks such as the contextual fear conditioning, that are strongly dependent on new neurons (Wojtowicz et al., 2008)are yet to be done. To establish such correlations one needs to modulate neurogenesis either further down or up toward the levels of the young animals. Hence, we asked the question whether the DG in middle-aged animals can support higher than normal levels of neurogenesis.

Various types of injury in the brain can trigger neurogenesis (Cameron and McKay, 1999; Lichtenwalner and Parent, 2006; Shetty et al., 2010). A notable example of such injury is

Table 2 | Summary of immunohistochemical data comparing sham and $\mathbf{2 V O}$ animals at 10, 35, 90 days after the surgery. BrdU was injected on day 7 in all cases.

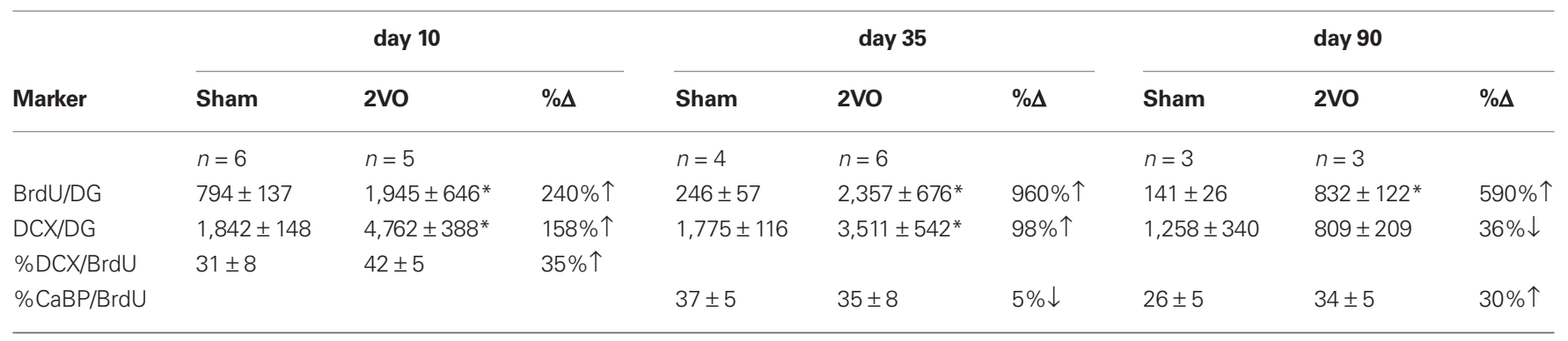

Mean areas $\left(\mu \mathrm{m}^{2}\right)$ and standard deviations are shown for each group. *indicates statistically significant difference between sham and 2 VO groups. 

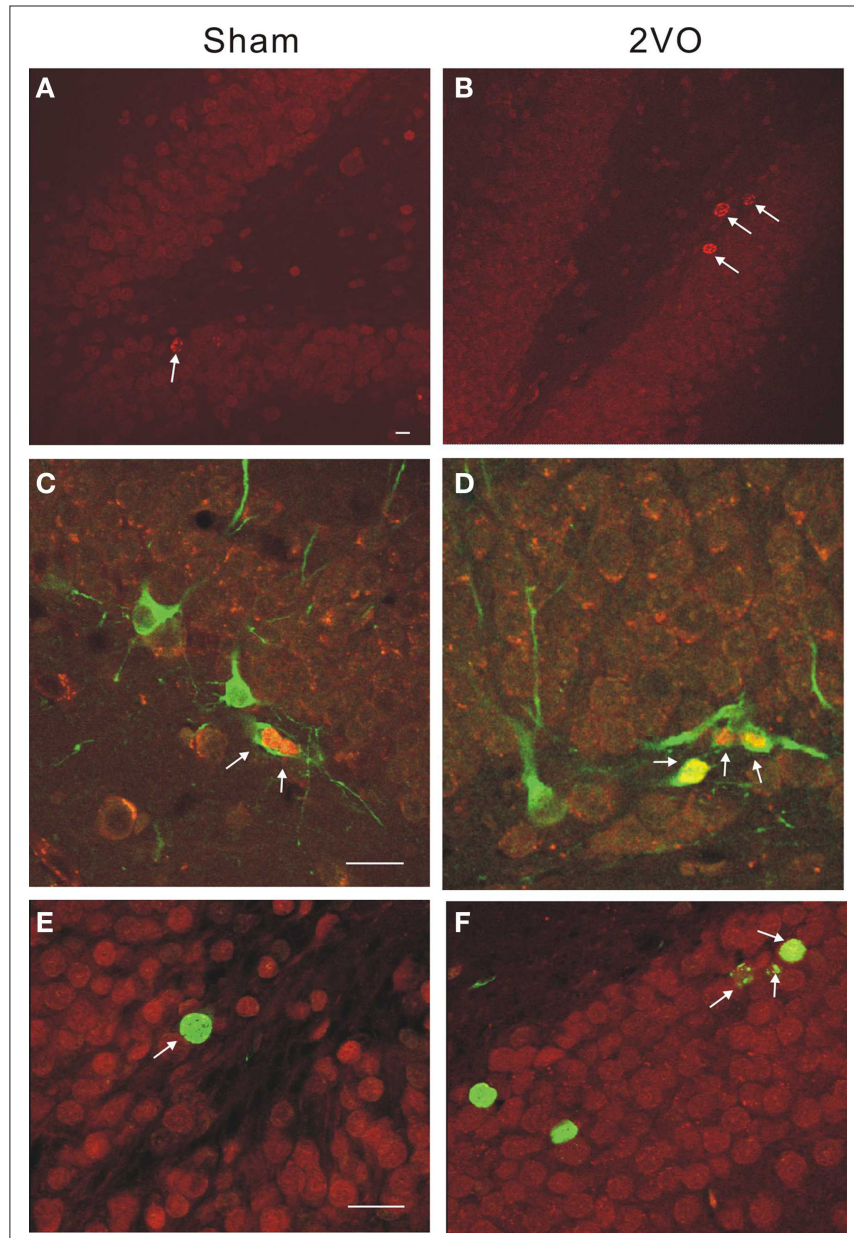

FIGURE 3 | Immunohistochemical staining illustrating neurogenesis in control and ischemic rats. (A,B) Single-labeled BrdU+ cells are more numerous (arrows) within a DG in $2 \mathrm{VO}$, ischemic animal at 10 days. (C,D) Double-labeled neuroblasts show BrdU+ (red) and DCX+ (green) neuroblasts at 10 days. (E,F) Double-labeled cells show BrdU+ (green) and $\mathrm{CaBP}+$ (red) neurons at 35 days. Calibration bar $=40$ microns in all cases.

experimental ischemia. Although most studies focus on ischemia as an experimental model of stroke our primary goal was to evaluate the neurogenic response and compare it to responses seen previously but in much younger rats. In one such study by Kee et al. (2001) the exact same experimental procedure induced an increase in neurogenesis by $50-100 \%$. This increase was transient and no longer significant at 35 days after ischemia even though some of the cells that were generated immediately after ischemia survived until day 35 (Wang et al., 2005). The study by Wang et al. (2005) further showed that changes in neurogenesis are truly compensatory since they correlated with electrophysiological changes in synaptic transmission and plasticity in DG. Elimination of neurogenesis with irradiation prevented this functional compensation (Wang et al., 2005).

Several other studies showed that both, focal and global anoxia can enhance neurogenesis (Lichtenwalner and Parent, 2006) in a predictable time-dependent manner, but most of the experiments have been done on relatively young mice and rats. The three stud- ies (Yagita et al., 2001; Jin et al., 2004; Darsalia et al., 2005) that used older animals produced varied results warranting further investigation. Our experiments agree qualitatively with those of (Yagita et al., 2001) who showed relatively higher post-ischemic neurogenic response in older animals, but with the use of alternative ischemia model (four vessel occlusion) and another rat strain (Wistar). Our study goes further in demonstrating enhanced neurogenesis on day 35 but not on day 90 after ischemia. This enhancement appears to be primarily due to increased proliferation, possibly by type 2 progenitors since it is reflected in the increase of DCX+ cells (Kempermann et al., 2004). This proliferation persists past day 10 and is responsible for a large overall increase in cell production on day 35 (see Figure 2). In contrast, proportional differentiation and consequent expression of DCX did not differ between control and ischemic animals (Table 2). Reduced proliferation of neural progenitors is a main characteristic of aging animals thus the exaggerated response seen after ischemia appears to be an appropriate compensation (McDonald and Wojtowicz, 2005).

In support of functional recovery, we also show that the typical polarization of neurogenesis along the dorso-ventral axis (Snyder et al., 2008, 2009b) of the hippocampus is significantly restored after ischemia. The functionality of these two hippocampal regions is polarized with the dorsal region being specialized for spatial activities and the ventral region for anxiety and odor-related activities (Bannerman et al., 2004; Pentkowski et al., 2006; Hunsaker et al., 2008). Since new neurons are thought to play a role in some of these behaviors, the recovery of the gradient may be an important aspect of the overall restoration of function.

In summary, our results are in line with previous reports showing that middle-aged animals retain limited ability to generate additional new neurons in response to physiological and pathological stimulation (Cameron and McKay, 1999; van Praag et al., 2005; Hattiangady et al., 2008; Rao et al., 2008; Shetty et al., 2010). Specifically, the results reported here indicate that enhancement of neurogenesis in middle-aged animals following injury can be achieved on temporary basis for more than 35 days but less than 90 days. One can speculate that more sustained effect could be obtained with additional trophic support. The list of transmitters and growth factors having influence over neurogenesis is extensive and it is likely that at least some are necessary to sustain neurogenesis at its normal rate (Drapeau and Abrous, 2008). Perhaps the most practical way to supply these factors to the ailing brain is by natural means, e.g., by exposing treated animals to enriched environment and or a training protocol. Cumulative evidence for enriched environment being beneficial for functional recovery after stroke is strong however the causal effect of newly produced neurons for this benefit has not yet been shown (Nithianantharajah and Hannan, 2006) and will require further experimentation.

\section{ACKNOWLEDGMENTS}

This work was supported by Heart and Stroke Foundation grant T5411 and a CIHR operating grant to J. Martin Wojtowicz. We thank Ms. Jaquelin Slinn for expertly performing $2 \mathrm{VO}$ and sham surgeries. 


\section{REFERENCES}

Bannerman, D. M., Rawlins, J. N., McHugh, S. B., Deacon, R. M., Yee, B. K., Bast, T., Zhang, W. N., Pothuizen, H. H., and Feldon, J. (2004). Regional dissociations within the hippocampus--memory and anxiety. Neurosci. Biobehav. Rev. 28, 273-283.

Bizon, J. L., and Gallagher, M. (2003). Production of new cells in the rat dentate gyrus over the lifespan: relation to cognitive decline. Eur. J. Neurosci. 18, 215-219.

Burke, S. N., and Barnes, C. A. (2006). Neural plasticity in the ageing brain. Nat. Rev. Neurosci. 7, 30-40.

Cameron, H. A., and McKay, R. D. G. (1999). Restoring production of hippocampal neurons in old age. Nat. Neurosci. 2, 894-897.

Darsalia, V., Heldmann, U., Lindvall, O., and Kokaia,Z. (2005). Stroke-induced neurogenesis in aged brain. Stroke 36, 1790-1795.

Deng, W., Aimone, J. B., and Gage, F. G. (2010). New neurons and new memories: How does adult hippocampal neurogenesis affect learning and memory? Nat. Neurosci. 11, 339-350.

Drapeau, E., and Abrous, D. N. (2008). Stem cell review series: role of neurogenesis in age-related memory disorders. Aging Cell 7, 569-589.

Drapeau, E., Mayo, W., Aurousseau, C., Le Moal, M., Piazza, P. V., and Abrous, D. N. (2003). Spatial memory performances of aged rats in the water maze predict levels of hippocampal neurogenesis. Proc. Natl. Acad. Sci. U.S.A. 100, 14385-14390.

Dupret, D., Revest, J. M., Koehl, M., Ichas, F., De Giorgi, F., Costet, P., Abrous, D. N., and Piazza, P. V. (2008). Spatial relational memory requires hippocampal adult neurogenesis. PLoS ONE 3, e1959. doi:10.1371/journal. pone.0001959.

Epp, J. R., Barker, J. M., and Galea, L. A. M. (2009). Running wild: Neurogenesis in the hippocampus across the lifespan in wild and laboratory-bred norway rats. Hippocampus 19, 1040-1049.

Hattiangady, B., Rao, M. S., and Shetty, A. K. (2008). Plasticity of hippocam$\mathrm{pal} \mathrm{stem/progenitor} \mathrm{cells} \mathrm{to} \mathrm{enhance}$ neurogenesis in response to kainateinduced injury is lost by middle age. Aging Cell 7, 207-224.

Hunsaker,M.R., Fieldsted,P.M., Rosenberg, J.S., and Kesner, R.P.(2008).Dissociating the roles of dorsal and ventral CA1 for the temporal processing of spatial locations, visual objects, and odors. Behav. Neurosci. 122, 643-650.

Imayoshi, I., Sakamoto, M., Ohtsuka, T., Takao, K., Miyakawa, T., Yamaguchi, M., Mori, K., Ikeda, T., Itohara, S., and Kageyama, R. (2008). Roles of continuous neurogenesis in the structural and functional integrity of the adult forebrain. Nat. Neurosci. 11, 1153-1161.

Jessberger, S., Clark, R. E., Broadbent, N., Clemenson, G. D. Jr., Consiglio, A., Lie, D. C., Squire, L. R., and Gage, F. G. (2009). Dentate gyrus-specific knockdown of adult neurogenesis impairs spatial and object recognition memory in adult rats. Learn. Mem. 16, 147-154.

Jin, J., Minami, M., Xie, L., Sun, Y., Mao, X. O., Wang, Y., Simon, R. P., and Greenberg, D. A. (2004). Ischemiainduced neurogenesis is preserved but reduced in the aged rodent brain. Aging Cell 3, 373-377.

Johnson, K. M., Boonstra, R., and Wojtowicz,J.M. (2010). Hippocampal neurogenesis in food-storing squirrels: the impact of age and spatial behavior. Genes Brain Behav. 9, 583-591.

Kee, N., Preston, E., and Wojtowicz, J. M. (2001). Enhanced neurogenesis after transient ischemia in the dentate gyrus of the rat. Exp. Brain Res. 136, 313-320.

Kempermann, G., Gast, D., and Gage, F. G. (2002). Neuroplasticity in old age: sustained fivefold induction of hippocampal neurogenesis by long term environmental enrichement. Ann. Neurol. 52, 135-143.

Kempermann, G., Jessberger, S., Steiner B., and Kronenberg, G. (2004) Milestones of neuronal development in the adult hippocampus. Trends Neurosci. 27, 446-452.

Kempermann, G., Kuhn, H. G., and Gage, F. H. (1998). Experience-induced neurogenesis in the senescent dentate gyrus. J. Neurosci. 18, 3206-3212.

Kokaia, Z., and Lindvall, O. (2003). Neurogenesis after ischaemic brain insults. Curr. Opin. Neurobiol. 13, 127-132.

Leuner, B., Gould, E., and Shors, T. J. (2006). Is there a link between adult neurogenesis and learning? Hippocampus 16, 216-224.

Lichtenwalner, R. J., and Parent, J. M. (2006). Adult neurogenesis and the ischemic forebrain. J. Cereb. Blood Flow Metab. 26, 1-20.

Liu, J., Solway, K., Messing, R. O., and Sharp, F. R. (1998). Increased neurogenesis in the dentate gyrus after transient global ischemia in Gerbils. J. Neurosci. 18, 7768-7778.

McDonald, H. Y., and Wojtowicz, J. M. (2005). Dynamics of neurogenesis in the dentate gyrus of adult rats. Neurosci. Lett. 385, 70-75.

Merrill, D. A., Karim, R., Darraq, M., Chiba, A. A., and Tuszynski, M. H (2003). Hippocampal cell genesis does not correlate with spatial learning ability in aged rats. J. Comp. Neurol. 459, 201-207.
Nithianantharajah, J., and Hannan, A. J. (2006). Enriched environments, experience-dependent plasticity and disorders of the nervous system. Nat Rev. Neurosci. 7, 697-709.

Pentkowski, N. S., Blanchard, D. C., Lever C., Litvin, Y., and Blanchard, R. J. (2006). Effects of lesions to the dorsal and ventral hippocampus on defensive behaviors in rats. Eur. J. Neurosci. 23 2185-2196.

Preston, E., and Webster, J. (2004). A two-hour window for hypothermic modulation of early events that impact delayed opening of the rat blood-brain barrier after ischemia. Acta Neurpathol. 108, 406-412.

Rao, M. S., Hattiangady, B., and Shetty, A. K. (2006). The window and mechanisms of major age-related decline in the production of new neurons within the dentate gyrus of the hippocampus. Aging Cell 5, 545-558.

Rao, M. S., Hattiangady, B., and Shetty, A. K. (2008). Status epilepticus during old age is not associated with enhanced hippocampal neurogenesis. Hippocampus 18, 931-944.

Schmued, L. C., and Hopkins, K. J. (2000) Fluoro-Jade B: a high affinity fluorescent marker for the localization of neuronal degeneration. Brain Res. 874, 123-130.

Sharp, F. R., Liu, J., and Bernabeu, R (2002). Neurogenesis following brain ischemia. Dev. Brain Res. 134 23-30.

Shetty, A. K., Hattiangady, B., Rao, M. S., and Shuai, B. (2010). Deafferentation enhances neurogenesisin theyoung and middle aged hippocampus but not in the aged hippocampus. Hippocampus doi: 10.1002/hipo.20776.

Small, S. A., Chawla, M. K., Buonocore, M., Rapp, P. R., and Barnes, C. A (2004). Imaging correlates of brain function in monkeys and rats isolates a hippocampal subregion differentially. Proc. Natl. Acad. Sci. U.S.A. 101 7181-7186.

Smith, M. L., Auer, R. N., and Siesjo, B. K (1984). The density and distribution of ischemic brain injury in the rat following 2-10 min of forebrain ischemia. Acta Neuropathol. 64, 319-332.

Snyder, J. S., Choe, J., Clifford, M., Jeurling, S. I., Hurley, P., Kamhi, J. F., and Cameron, H. A. (2009a). Adultborn hippocampal neurons are more numerous, faster maturing and more involved in behavior in rats than in mice. J. Neurosci. 29, 14484-11445.

Snyder, J. S., Radik, R., Wojtowicz, J. M., and Cameron, A. M. (2009b) Septo-temporal gradients of neurogenesis and activity in 13 months-old rats. Neurobiol. Aging doi: 10.1016/j. neurobiolaging.2009.05.022. [Epub ahead of print].
Snyder, J. S., Radik, R., Wojtowicz, J. M., and Cameron, H. A. (2008). Anatomical gradients of neurogenesis and activity: young neurons in the ventral dentate gyrus are activated by water maze training. Hippocampus 19, 360-370.

van Praag, H., Shubert, T., Zhao, C., and Gage, F. G. (2005). Exercise enhances learning and hippocampal neurogenesis in aged mice. J. Neurosci. 28, 8680-8685.

Wang, S., Kee, N., Preston, E. and Wojtowicz, J. M. (2005). Electrophysiological correlates of neural plasticity compensating for ischemia-induced damage in the hippocampus. Exp. Brain Res. 165, 250-260.

West, M. J.,Slomianka, L., and Gundersen, H. J. G. (1991). Unbiased stereological estimation of the total number of neurons in the subdivisions of the rat hippocampus using the optical fractinator. Anat. Rec. 231, 482-497.

Wojtowicz, J. M. (2008). Potential consequences of altered neurogenesis on learning and memory in the epileptic brain. Epilepsia 49(Suppl. 5), 42-49.

Wojtowicz, J. M., Askew, M. L., and Winocur, G. (2008). The effects of running and inhibiting adult neurogenesis on learning and memory in rats. Eur. J. Neurosci. 27, 1494-1502.

Wojtowicz, J. M., and Kee, N. (2006). BrdU assay for neurogenesis in rodents. Nat. Protoc. 1, 1399-1405.

Yagita, Y., Kitagawa, K., Ohtsuki, T. Takasawa, K., Miyata, T., Okano, H., Hori, M., and Matsumoto, M. (2001). Neurogenesis by progenitor cells in the ischemic adult rat hippocampus. Stroke 32, 1890-1896.

Conflict of Interest Statement: The authors declare that the research was conducted in the absence of any commercial or financial relationships that could be construed as a potential conflict of interest.

Received: 12 July 2010; paperpending published: 28 July 2010; accepted: 16 August 2010; published online: 30 August 2010.

Citation: Tan Y-F, Preston E and Wojtowicz JM (2010) Enhanced post-ischemic neurogenesis in aging rats. Front. Neurosci. 4:163. doi:10.3389/fnins.2010.00163.

This article was submitted to Frontiers in Neurogenesis, a specialty of Frontiers in Neuroscience.

Copyright (c) 2010 Tan, Preston and Wojtowicz. This is an open-access article subject to an exclusive license agreement between the authors and the Frontiers Research Foundation, which permits unrestricted use, distribution, and reproduction in any medium, provided the original authors and source are credited. 